\title{
Consensus of the Distributed Varying Scale Wireless Sensor Networks
}

\author{
Jinping Mou, ${ }^{1,2}$ Wuneng Zhou, ${ }^{1}$ Tianbo Wang, ${ }^{1}$ Chuan Ji, ${ }^{1}$ and Dongbing Tong ${ }^{1}$ \\ ${ }^{1}$ College of Information Sciences and Technology, Donghua University, Shanghai 200051, China \\ ${ }^{2}$ College of Information Sciences and Mathematics, Taizhou University, Zhejiang 317000, China
}

Correspondence should be addressed to Jinping Mou; mjptougaozhuanyong@163.com

Received 21 September 2012; Accepted 22 January 2013

Academic Editor: Xinzhu Meng

Copyright (C) 2013 Jinping Mou et al. This is an open access article distributed under the Creative Commons Attribution License, which permits unrestricted use, distribution, and reproduction in any medium, provided the original work is properly cited.

Consensus problems are investigated for a type of the distributed varying scale wireless sensor network (VSWSN), where the scale of the network is increasing or decreasing due to the new nodes joining in or the invalid nodes quitting from WSN, respectively. In order to demonstrate the communicating behaviors more realistic, we offer the node attached component sequence for each valid node under the general sleep algorithm. Based on the component sequences, several concepts, such as the local limited intersection connection (LLI connection) and the global limited intersection connection (GLI connection), are provided to display the connectivity of the varying topology. Under certain conditions, the designed protocol ensures that all sensors arrive at the component consensus or the global consensus if the communicating graph is LLI connected or GLI connected, respectively. By the basic theoretical analysis, several consensus criterions are obtained. In the meanwhile, the consensus regions are investigated. The numerical example shows the reliability of the proposed results.

\section{Introduction}

Consensus is of great importance in many applications of wireless sensor networks (WSNs) [1], such as the clock synchronization [2], data fusion $[3,4]$, and formation control [5]. To achieve the consensus, it requires that all nodes of the networks achieve the special value, while they may begin with the different initial values.

In the past few years, consensus problems have been widely investigated, and many exciting results have been obtained. The early work of consensus problems can be found in [6], where the consensus problems are investigated for the fixed scale networks. The most further researches focus on optimizing the algorithms and the communicating topology, such as in [7-10], and the optimal algorithms are taken to solve the consensus. Other researchers devote to optimize the topology, such as Lin and Jia solve the average consensus problems of multiagents with time delay in the jointly connected topology [11]; Atrianfar and Haeri deal with the average consensus problems in the weak connected topology [12]; consensus problems are studied under the sleeping-awaking method $[13,14]$.
In recent years, some consensus results of the scale-free networks, where the scale of the networks may be increasing, are obtained in the exciting literatures $[15,16]$.

And so far, consensus regions of the networks have attracted much attention by some researchers, such as Li et al. who propose an observer-type protocol to solve the consensus problem, and meanwhile an unbounded consensus region was provided [17]. Based on the connected network, consensus regions are investigated in the multiagent dynamical systems $[18,19]$.

It should point out that in the traditional consensus literatures, the scale of the network is fixed or increasing. Namely, the newly joined nodes and the invalid removed nodes have not been considered. In fact, the varying scale wireless sensor network (VSWSN) exists in many applications. For example, after the limited power exhausted, the nodes become invalid, or when the new nodes join the network, the scale of WSN is changed, and the related consensus regions are indefinite. However, up to now, consensus problems of VSWSN with the consensus regions have not caused much attention.

The main purpose of this paper is to investigate the consensus problems of VSWSN and its consensus regions. In 
the traditional literatures, the connected topology, the weak connected topology, or the jointly connected topology is the basic condition for the network communication. Different from the traditional literatures, in VSWSN, while some new nodes join in the network and some nodes quit from the network, VSWSN may neither connected, nor jointly connected, nor weak connected, and the communicating behaviors among those nodes are indefinite and random. The communicating graph of VSWSN which we proposed in this paper is displayed by the node attached component sequence. It turns out that if every two nodes have chance to connect, namely, the topology is the local limited intersection connected (LLI connected) or the global limited intersection connected (GLI connected), and the intersection topology has the enough dwell time, then under the designed protocol, VSWSN achieves the component consensus or the global consensus.

Moreover, the consensus regions are different from the traditional literatures. In the traditional literatures, the consensus value is determined by the state of the fixed node set. In VSWSN, the consensus value is varying according to the varying node set. So the considered systems in this paper are more general than that of network with the fixed node set, which the topology is the special case of the global limited intersection connection.

The outline of this paper is listed as follows. In Section 2, some basic concepts and notations are introduced. In Section 3, we provided the main results. For each certain topology, the consensus criterions and the consensus regions are investigated in detail. For the varying topology in which some nodes may be added while some other nodes may be removed, the consensus criterions and the consensus regions are also provided. In Section 5, a numeral example shows the reliability of the proposed results. In Section 6, several conclusions are obtained.

\section{Preliminaries}

2.1. Graph Theory and Notations. Let $\aleph=\{\overline{0}, \overline{1}, \overline{2}, \ldots, \bar{\kappa}$, $\overline{\kappa+1}, \ldots\}$ be all possible topologies of WSN, where the relations of the elements can be expressed by the partial sequence $\overline{0} \preceq \overline{1} \preceq \overline{2} \preceq \cdots \preceq \bar{\kappa} \preceq \cdots$, and $\overline{0}$ is the initial topology.

Let $\theta: T \rightarrow \aleph$ be the discernible function among the topologies, $\theta(k)=\bar{\kappa} \in \aleph$, where $\left(\widetilde{m}_{\kappa-1}+1\right) l \leq k \leq \widetilde{m}_{\kappa} l$, $l \in R^{+}, k=z l, z \in N, T=\left\{0, l, 2 l, \ldots, \widetilde{m}_{0} l,\left(\widetilde{m}_{0}+1\right) l, \ldots\right.$, $\left.\widetilde{m}_{1} l, \ldots, \widetilde{m}_{\kappa} l, \ldots\right\}, \widetilde{m}_{\kappa}=m_{0}+m_{1}+\cdots+m_{\kappa},\left\{m_{0}, m_{1}, \ldots\right.$, $\left.m_{\kappa}, \ldots\right\} \subseteq Z^{+}$, and $m_{\kappa}$ is called the dwell time.

According to the function $\theta$, the varying scale WSN (VSWSN) can be denoted by a varying graph $G\left(\theta\left(\widetilde{m}_{\kappa} l\right)\right)=$ $G(\bar{\kappa})=\{V(\bar{\kappa}), A(\bar{\kappa}), E(\bar{\kappa})\}$, where $V(\bar{\kappa})=V_{1}(\bar{\kappa}) \cup V_{2}(\bar{\kappa})$, $V_{1}(\bar{\kappa})=\left\{1_{\bar{\kappa}}, 2_{\bar{\kappa}}, \ldots, i_{\bar{\kappa}}, \ldots, u_{\bar{\kappa}}\right\}$ is the valid node set that all elements come from the former topology $\overline{\kappa-1}, V_{2}(\bar{\kappa})=\left\{1_{\bar{\kappa}}^{\prime}\right.$, $\left.2_{\overline{\mathcal{K}}}^{\prime}, \ldots, v_{\overline{\mathcal{K}}}^{\prime}\right\}$ is the newly joined node set, and $E(\bar{\kappa})=\left\{\left(i_{\bar{\kappa}}, j_{\bar{\kappa}}\right) \mid\right.$ $\left.i_{\bar{\kappa}} \neq j_{\bar{\kappa}}, i_{\bar{\kappa}}, j_{\bar{\kappa}} \in V(\bar{\kappa})\right\}$ is the edge set. $A(\bar{\kappa})=\left(a_{i j}(\bar{\kappa})\right)_{w_{\bar{\kappa}}} \in$ $R^{w_{\bar{\kappa}} \times w_{\bar{\kappa}}}$ is the weighted symmetric matrix, where $w_{\bar{\kappa}}=u_{\bar{\kappa}}+v_{\bar{\kappa}}$, if $a_{i j}(\bar{\kappa})=1$, then there exists information flow between $i_{\bar{\kappa}}$ and $j_{\bar{\kappa}}$, if $a_{i j}(\bar{\kappa})=0$, then there is no communication between $i_{\bar{\kappa}}$ and $j_{\bar{\kappa}} . N_{i_{\bar{\kappa}}}=\left\{j_{\bar{\kappa}} \mid i_{\bar{\kappa}} \neq j_{\bar{\kappa}}, i_{\bar{\kappa}}, j_{\bar{\kappa}} \in V(\bar{\kappa})\right\}$ refers to the neighbor set of node $i_{\bar{\kappa}}$ in the topology $\bar{\kappa}$.

$L\left(w_{\bar{\kappa}}\right)=\left(l_{i j}(\bar{\kappa})\right)_{w_{\bar{\kappa}} \times w_{\bar{\kappa}}}$ is a Laplacian matrix, and $l_{i_{\bar{\kappa}} j_{\bar{\kappa}}}$ is defined as

$$
l_{i j}(\overline{\mathcal{\kappa}})= \begin{cases}\sum_{j_{\overline{\mathcal{K}}}} a_{i j}(\overline{\mathcal{K}}), & i_{\overline{\mathcal{K}}}=j_{\overline{\mathcal{K}}}, j_{\overline{\mathcal{K}}} \in N_{i_{\overline{\mathcal{\kappa}}}}(k), \\ -a_{i j}(\overline{\mathcal{\kappa}}), & i_{\bar{\kappa}} \neq j_{\overline{\mathcal{K}}}, j_{\overline{\mathcal{K}}} \in N_{i_{\overline{\mathcal{K}}}}(k) .\end{cases}
$$

Similarly, a component which the connected subgraph of $G(\bar{\kappa})$ can be denoted by $C^{\prime}(\bar{\kappa})=\left\{V^{\prime}(\bar{\kappa}), A^{\prime}(\bar{\kappa}), E^{\prime}(\bar{\kappa})\right\}, V^{\prime}(\bar{\kappa}) \subset$ $V(\bar{\kappa}), E^{\prime}(\bar{\kappa}) \subset E(\bar{\kappa})$. The relation between $G(\bar{\kappa})$ and $C^{\prime}(\bar{\kappa})$ is denoted by $C^{\prime}(\bar{\kappa}) \subset G(\bar{\kappa})$.

Definition 1. For all $i_{\bar{\kappa}} \in V(\bar{\kappa})$, if there exists a component $C^{\prime}(\bar{\kappa})$ of $G(\bar{\kappa})$ such that $i_{\bar{\kappa}} \in V^{\prime}(\bar{\kappa})$, then $C^{\prime}(\bar{\kappa})$, is said to be the node attached component of $i_{\bar{\kappa}}$, if there exists the sequence $C^{\prime}(\bar{\kappa}), C^{\prime}(\overline{\kappa+1}), \ldots$, such that $i_{\bar{\kappa}} \in V^{\prime}(\bar{\kappa}), i_{\bar{\kappa}} \in V^{\prime}(\overline{\kappa+1}) \ldots$, then the sequence $C^{\prime}(\bar{\kappa}), C^{\prime}(\overline{\kappa+1}), \ldots$ is said to be the node attached component sequence of node $i_{\bar{\kappa}}$.

Definition 2. For all $i_{\bar{\kappa}}, j_{\bar{\kappa}} \in V(\bar{\kappa})$, if there exists two related attached component sequences $C^{\prime}\left(\bar{\kappa}_{1}\right), C^{\prime}\left(\bar{\kappa}_{2}\right), \ldots$, $C^{\prime}\left(\bar{\kappa}_{r}\right), \ldots$, and $C^{\prime \prime}\left(\bar{\kappa}_{1}\right), C^{\prime \prime}\left(\bar{\kappa}_{2}\right), \ldots, C^{\prime \prime}\left(\bar{\kappa}_{r}\right), \ldots$, respectively, and if there exist $\bar{\kappa}_{0} \in \aleph$ and $\aleph^{\prime}=\left\{\bar{\kappa}_{r_{1}}, \bar{\kappa}_{r_{1}+1}, \ldots, \bar{\kappa}_{r_{1}+m_{1}}\right.$, $\left.\bar{\kappa}_{r_{2}+1}, \ldots, \bar{\kappa}_{r_{2}+m_{2}}, \ldots, \bar{\kappa}_{r_{q}+1}, \bar{\kappa}_{r_{q}+m_{q}}, \ldots\right\} \subset \aleph$, such that

$$
\begin{gathered}
C^{\prime}\left(\bar{\kappa}_{r_{1}+m_{1}}\right)=C^{\prime \prime}\left(\bar{\kappa}_{r_{1}+m_{1}}\right), \\
C^{\prime}\left(\bar{\kappa}_{r_{2}+m_{2}}\right)=C^{\prime \prime}\left(\bar{\kappa}_{r_{2}+m_{2}}\right), \ldots, \\
C^{\prime}\left(\bar{\kappa}_{r_{q}+m_{q}}\right)=C^{\prime \prime}\left(\bar{\kappa}_{r_{q}+m_{q}}\right), \ldots,
\end{gathered}
$$

where $r_{1}, m_{1}, \ldots, r_{q}, m_{q} \in Z^{+}, \kappa_{0} \preceq \bar{\kappa}_{r_{1}} \preceq \bar{\kappa}_{r_{1}+1} \preceq \cdots \preceq$ $\bar{\kappa}_{r_{q}+m_{q}}$, then the communicating graph is said to be the local limited intersection connected (LLI connected), and $\bar{\kappa}_{r_{1}+m_{1}}, \bar{\kappa}_{r_{2}+m_{2}}, \ldots, \bar{\kappa}_{r_{q}+m_{q}}$ are called the intersection topologies.

Definition 3. For all $i_{\bar{\kappa}}, j_{\overline{\mathcal{K}}} \in V(\overline{\mathcal{\kappa}})$, if there exists two related attached component sequences $C^{\prime}\left(\bar{\kappa}_{1}\right), C^{\prime}\left(\bar{\kappa}_{1}\right), \ldots, C^{\prime}\left(\bar{\kappa}_{r}\right), \ldots$ and $C^{\prime \prime}\left(\bar{\kappa}_{1}\right), C^{\prime \prime}\left(\bar{\kappa}_{1}\right), \ldots, C^{\prime \prime}\left(\bar{\kappa}_{r}\right), \ldots$, respectively, and if there exist $\bar{\kappa}_{0} \in \aleph$ and $\aleph^{\prime}=\left\{\bar{\kappa}_{r}, \bar{\kappa}_{r+1}, \ldots\right\} \subset \aleph$, such that for all $\bar{\kappa}_{0} \preceq \bar{\kappa}_{r}, C^{\prime}\left(\bar{\kappa}_{r}\right)=C^{\prime \prime}\left(\bar{\kappa}_{r}\right)$, then the communicating graph is said to be the global limited intersection connected (GLI connected).

Under LLI connected and GLI connected, we are going to discuss the consensus problems of VSWSN in the following sections.

2.2. Problem Statement. In many applications, the communication of VSWSN is based on the multiple components. In this paper, the consensus problems which we considered are the component-based communication.

For $i_{\bar{\kappa}} \in V(\bar{\kappa})$, let $x_{i}(k, \bar{\kappa})$ be the state of sensor or node $i_{\bar{\kappa}}$, where $x_{i}(k, \bar{\kappa}) \in R^{d}, d \in Z^{+}$. Suppose the state of $i_{\bar{\kappa}}$ is given by

$$
x_{i}(k+l, \bar{\kappa})=b x_{i}(k, \bar{\kappa})+u_{i_{\bar{\kappa}}}(k) \text {, }
$$


where $b$ is a constant, $u_{i_{\bar{\kappa}}}(k)$ is the consensus protocol and is given by

$$
u_{i_{\bar{\kappa}}}(k)=\epsilon_{i_{\bar{\kappa}}} \sum_{j_{\bar{\kappa}} \in N_{i_{\bar{\kappa}}}(k)}\left[a_{i j}(\bar{\kappa}) x_{j}(k, \bar{\kappa})-\tilde{a}_{i j}(\bar{\kappa}) x_{i}(k, \bar{\kappa})\right],
$$

where $\tilde{a}_{i j}(\bar{\kappa})$ is a positive constant.

For all $C^{\prime}(\bar{\kappa}) \subset G(\bar{\kappa})$, under the dynamic (3) and protocol (4), the state of component $C^{\prime}(\bar{\kappa})$ which attaches on node $i_{\bar{\kappa}}$ can be displayed as follows:

$$
X_{\widehat{n}_{\bar{\kappa}}}(k+l)=\left(B_{\widehat{n}_{\bar{\kappa}}}-\epsilon_{\widehat{n}_{\bar{\kappa}}} L_{\widehat{n}_{\bar{\kappa}}}\right) X_{\widehat{n}_{\bar{\kappa}}}(k),
$$

where

$$
\begin{gathered}
\left(\widetilde{m}_{\kappa-1}+1\right) l \leq k \leq \widetilde{m}_{\kappa} l, \quad \widehat{n}_{\bar{\kappa}}=p_{\bar{\kappa}}+q_{\bar{\kappa}}^{\prime} \leq w_{\bar{\kappa}}, \\
B_{\widehat{n}_{\bar{\kappa}}}=\operatorname{diag}\{b, \ldots, b, \ldots, b\}, \\
\epsilon_{\widehat{n}_{\bar{\kappa}}}=\operatorname{diag}\left\{\epsilon_{1_{\bar{\kappa}}}, \ldots, \epsilon_{i_{\bar{\kappa}}}, \ldots, \epsilon_{\widehat{n}_{\bar{\kappa}}}\right\} .
\end{gathered}
$$

$L_{\widehat{n}_{\bar{\kappa}}}=\left(l_{i_{\bar{\kappa}} j_{\bar{\kappa}}}\right)_{\widehat{n}_{\bar{\kappa}} \times \widehat{n}_{\bar{\kappa}}}$, and $l_{i_{\bar{\kappa}} j_{\bar{\kappa}}}$ is defined as

$$
\begin{aligned}
& l_{i_{\bar{\kappa}} j_{\bar{\kappa}}}= \begin{cases}\sum_{j_{\bar{\kappa}}} \widetilde{a}_{i j}(\overline{\mathcal{K}}), & i_{\overline{\mathcal{\kappa}}}=j_{\overline{\mathcal{K}}}, j_{\overline{\mathcal{K}}} \in N_{i_{\bar{\kappa}}}(k), \\
-a_{i j}(\overline{\mathcal{K}}), & i_{\overline{\mathcal{\kappa}}} \neq j_{\overline{\mathcal{K}}}, j_{\overline{\mathcal{K}}} \in N_{i_{\bar{\kappa}}}(k),\end{cases} \\
& X_{\widehat{n}_{\bar{\kappa}}}(k)=\left[X_{p_{\bar{\kappa}}}(k)^{T}, X_{q_{\bar{\kappa}}^{\prime}}(k)^{T}\right]^{T} \text {, }
\end{aligned}
$$

$X_{p_{\bar{\kappa}}}(k)$ is the vector state of the valid nodes that come from the former topology $\overline{\kappa-1}, X_{q_{\bar{\kappa}}^{\prime}}(k)$ is the vector state of the newly joined nodes, $p_{\overline{\mathcal{\kappa}}} \leq u_{\overline{\mathcal{K}}}, q_{\overline{\mathcal{\kappa}}}^{\prime} \leq v_{\overline{\mathcal{K}}}$,

$$
\begin{array}{r}
X_{p_{\bar{\kappa}}}(k)=\left[x_{1_{\bar{\kappa}}}(k, \bar{\kappa}), \ldots, x_{i}(k, \bar{\kappa}), \ldots, x_{p}(k, \bar{\kappa})\right]^{T}, \\
i_{\bar{\kappa}} \in V_{1}(\bar{\kappa}), \\
X_{q_{\bar{\kappa}}^{\prime}}(k)=\left[x_{1^{\prime}}(k, \bar{\kappa}), \ldots, x_{i^{\prime}}(k, \bar{\kappa}), \ldots, x_{q^{\prime}}(k, \bar{\kappa})\right]^{T}, \\
i_{\bar{\kappa}}^{\prime} \in V_{2}(\bar{\kappa}) .
\end{array}
$$

Remark 4. Analogously, if $\widehat{n}_{\bar{\kappa}}$ is substituted by $w_{\bar{\kappa}}$, then system (5) expresses the state of the whole VSWSN.

For the node attached component $C^{\prime}(\bar{\kappa})$, sensors $1_{\bar{\kappa}}^{\prime}$, $\ldots, q_{\bar{\kappa}}^{\prime}$ are the newly joined nodes, and the state of each newly joined node at time $k$ is equal to the state at time $k-l$ in the topology $\overline{\kappa-1}$.

Definition 5. For all $C^{\prime}(\bar{\kappa}) \subset G(\bar{\kappa})$, if

$$
\begin{aligned}
& \sum_{i_{\bar{\kappa}}, j_{\bar{\kappa}} \in V^{\prime}(\bar{\kappa})}\left\|x_{i}\left(\widetilde{m}_{\kappa} l, \bar{\kappa}\right)-x_{j}\left(\widetilde{m}_{\kappa} l, \bar{\kappa}\right)\right\|^{2} \\
& \quad<c^{m_{\kappa}} \widetilde{d}_{i_{\bar{\kappa}}} \sum_{i_{\bar{\kappa}} \in V^{\prime}(\bar{\kappa})}\left\|x_{i}\left(\left(\widetilde{m}_{\kappa-1}+1\right) l, \bar{\kappa}\right)\right\|^{2},
\end{aligned}
$$

where $0<c<1, \widetilde{d}_{i_{\bar{\kappa}}}$ refers to the maximal degree node of $C^{\prime}(\bar{\kappa})$, then VSWSN (5) is said to be the component potential consensus. In addition, if $C^{\prime}(\bar{\kappa})=G(\bar{\kappa})$ and (9) holds, then VSWSN (5) is said to be the global potential consensus.
Definition 6. For all $C^{\prime}(\bar{\kappa}) \subset G(\bar{\kappa})$ and for all $i_{\bar{\kappa}}, q_{\bar{\kappa}} \in V^{\prime}(\bar{\kappa})$, if

$$
\lim _{k \rightarrow \infty}\left\|x_{i}(k, \bar{\kappa})-x_{q}(k, \bar{\kappa})\right\|=0,
$$

then VSWSN (5) is said to be achieved the component consensus. In addition, if $C^{\prime}(\bar{\kappa})=G(\bar{\kappa})$ and (10) holds, then VSWSN (5) is said to be achieved the global consensus.

Let $x_{i}^{0}(k, \bar{\kappa})=\left(1 / \widehat{n}_{\bar{\kappa}}\right) \sum_{i_{\bar{\kappa}} \in V^{\prime}(\bar{\kappa})} x_{i}(k, \bar{\kappa})$, if $\lim _{k \rightarrow \infty} \| x_{i}(k, \bar{\kappa})-$ $x_{i}^{0}(k, \bar{\kappa}) \|=0$, then VSWSN (5) is said to be achieved the component average consensus. In addition, if $C^{\prime}(\bar{\kappa})=G(\bar{\kappa})$ and (10) holds, then VSWSN (5) is said to be achieved the global average consensus.

Theorem 7. For all $C^{\prime}(\bar{\kappa}) \subset G(\bar{\kappa})$, if there exists a constant $\eta$, such that

$$
\left[\left(B_{\widehat{n}_{\bar{\kappa}}}-\epsilon_{\widehat{n}_{\bar{\kappa}}} L_{\widehat{n}_{\bar{\kappa}}}\right)^{T}\right]^{m_{\kappa}} L\left(\widehat{n}_{\bar{\kappa}}\right)\left(B_{\widehat{n}_{\bar{\kappa}}}-\epsilon_{\widehat{n}_{\bar{\kappa}}} L_{\widehat{n}_{\bar{\kappa}}}\right)^{m_{\kappa}}<\widetilde{d}_{i_{\bar{\kappa}}} \eta^{2 m_{\kappa}} I_{\widehat{n}_{\bar{\kappa}}}
$$

where $0<\eta<1$, then VSWSN (5) is the component potential consensus. In addition, if $C^{\prime}(\bar{\kappa})=G(\bar{\kappa})$, then VSWSN (5) is the global potential consensus.

Proof. Note that $\sum_{i_{\bar{\kappa}}, q_{\bar{\kappa}}^{\prime} \in V^{\prime}(\bar{\kappa})}\left\|x_{i}\left(\widetilde{m}_{\kappa} l, \bar{\kappa}\right)-x_{j}\left(\widetilde{m}_{\kappa} l, \bar{\kappa}\right)\right\|^{2}=$ $X_{\widehat{n}_{\bar{\kappa}}}^{T}\left(\widetilde{m}_{\kappa} l\right) L\left(\widehat{n}_{\bar{\kappa}}\right) X_{\widehat{n}_{\bar{\kappa}}}\left(\widetilde{m}_{\kappa} l\right)=X_{\widehat{n}_{\bar{\kappa}}}^{T}\left(\left(\widetilde{m}_{\kappa-1}+1\right) l\right)\left[\left(B_{\widehat{n}_{\bar{\kappa}}}-\right.\right.$ $\left.\left.\epsilon_{\widehat{n}_{\bar{\kappa}}} L_{\widehat{n}_{\bar{\kappa}}}\right)^{T}\right]^{m_{\kappa}} L\left(\widehat{n}_{\bar{\kappa}}\right)\left(B_{\widehat{n}_{\bar{\kappa}}}-\epsilon_{\widehat{n}_{\bar{\kappa}}} L_{\widehat{n}_{\bar{\kappa}}}\right)^{m_{\kappa}} X_{\widehat{n}_{\bar{\kappa}}}\left(\left(\widetilde{m}_{\kappa-1}+1\right) l\right)$, if $\left[\left(B_{\widehat{n}_{\bar{\kappa}}}-\epsilon_{\widehat{n}_{\bar{\kappa}}} L_{\widehat{n}_{\bar{\kappa}}}\right)^{T}\right]^{m_{\kappa}} L\left(\widehat{n}_{\bar{\kappa}}\right)\left(B_{\widehat{n}_{\bar{\kappa}}}-\epsilon_{\widehat{n}_{\bar{\kappa}}} L_{\widehat{n}_{\bar{\kappa}}}\right)^{m_{\kappa}}<\widetilde{d}_{i_{\bar{\kappa}}} \eta^{2 m_{\kappa}} I_{\widehat{n}_{\bar{\kappa}}}$, then

$$
\begin{gathered}
\sum_{i_{\bar{\kappa}}, q_{\bar{\kappa}}^{\prime} \in V^{\prime}(\bar{\kappa})}\left\|x_{i}\left(\widetilde{m}_{\kappa} l, \bar{\kappa}\right)-x_{j}\left(\widetilde{m}_{\kappa} l, \bar{\kappa}\right)\right\|^{2} \\
<\widetilde{d}_{i_{\bar{\kappa}}} \eta^{2 m_{\kappa}}\left\|X_{\widehat{n}_{\bar{\kappa}}}\left(\left(\widetilde{m}_{\kappa-1}+1\right) l\right)\right\|^{2} .
\end{gathered}
$$

Similarly, we can get the result when $C^{\prime}(\bar{\kappa})=G(\bar{\kappa})$.

Remark 8. If VSWSN (5) is the component potential consensus or the global potential consensus, then the errors among the adjacent nodes are decreased, but it does not mean that system achieves consensus, in any case, the consensus is constrained by the dwell time $m_{\kappa}$.

Assumption 1. We assume that for each intersection topology $\bar{\kappa}$, their exists $m>0$, such that $m_{\kappa}>m$.

Remark 9. If a sensor leaves from its neighbors and becomes an isolated node, and its state may not keep in coordination with other nodes. Note that if the communicating graph is LLI connected or GLI connected, the node has chance to keep in coordinating with other nodes.

For all $i_{\bar{\kappa}} \in V^{\prime}(\bar{\kappa})$, let $e_{\bar{\kappa}}^{i}(k)=x_{i}(k, \bar{\kappa})-x_{i}^{0}(k, \bar{\kappa})$; we get the systematic error of (5) as follows:

$$
\begin{aligned}
e_{\bar{\kappa}}(k) & =X_{\widehat{n}_{\bar{\kappa}}}(k)-\mathbf{1}_{\widehat{n}_{\bar{\kappa}}} \otimes x_{i}^{0}(k, \bar{\kappa})=\Gamma X_{\widehat{n}_{\bar{\kappa}}}(k) \\
& =\Gamma\left(B_{\widehat{n}_{\bar{\kappa}}}-L_{\widehat{n}_{\bar{\kappa}}}\right) X_{\widehat{n}_{\bar{\kappa}}}(k-l),
\end{aligned}
$$


where $e_{\bar{\kappa}}(k)=\left(e_{\overline{\mathcal{K}}}^{1}(k), e_{\overline{\mathcal{K}}}^{2}(k) \ldots, e_{\overline{\mathcal{K}}}^{\widehat{n}}(k)\right)^{T}, \Gamma=I_{\widehat{n}_{\bar{\kappa}}}-\left(1 / \widehat{n}_{\bar{\kappa}}\right) \times$ $\mathbf{1}_{\widehat{n}_{\bar{\kappa}} \times \widehat{n}_{\bar{\kappa}}}$, and $\mathbf{1}_{\widehat{n}_{\bar{\kappa}} \times \widehat{n}_{\bar{\kappa}}}$ is the $\widehat{n}_{\bar{\kappa}} \times \widehat{n}_{\overline{\mathcal{\kappa}}}$ matrix in which each entry is 1.

The primary purpose of this paper is to provide the basic consensus analysis of (5) for the aforementioned scenarios. Because the scale of the network is a variable, different from the traditional literatures which investigate the consensus problems are based on the global structure, to study consensus problems, we depend on the component sequences. In the meanwhile, the consensus regions are gotten in the following sections.

\section{Main Results}

In this section, we are going to discuss the component potential consensus and the global potential consensus firstly. Then the relations between the component potential consensus and the component consensus, the global potential consensus and the global consensus are studied.

3.1. Consensus Investigation for WSN in a Certain Topology. In this subsection, we study the potential consensus problems of the certain topology. For convenience, we focus on the component $C^{\prime}(\bar{\kappa})$, its topology index is dropped and denoted by $C^{\prime}$, and its state is denoted by $X_{\widehat{n}}(k)$. The error of $X_{\widehat{n}}(k)$ is denoted by $e(k)=X_{\widehat{n}}(k)-\mathbf{1}_{\widehat{n} \times 1} \otimes x_{i}^{0}(k)$. To express the state of $e(k)$, we depend on the eigenvalues. Let eigen $\left(B_{\widehat{n}}-\epsilon L_{\widehat{n}}\right)$ be the eigenvalue set of the matrix $\left(B_{\widehat{n}}-\epsilon L_{\widehat{n}}\right)$, let $\lambda_{i}$ be the $i$ th eigenvalue of $B_{\widehat{n}}-\epsilon L_{\widehat{n}}$, and $\lambda=\max \left\{\left|\lambda_{i}\right| \mid i \in V^{\prime}(\bar{\kappa})\right\}$.

To achieve the potential consensus, it requires that the error

$$
\begin{aligned}
e(k) & =X_{\widehat{n}}(k)-\mathbf{1}_{\widehat{n} \times 1} \otimes x_{i}^{0}(k)=\Gamma X_{\widehat{n}}(k) \\
& =\Gamma\left(B_{\widehat{n}}-\epsilon L_{\widehat{n}}\right) X_{\widehat{n}}(k-l)
\end{aligned}
$$

is decreasing. To keep the decreasing error, it requires that $\lambda$ takes in some values, and the following discussion focuses on how to take the value of parameter $\epsilon_{i}$ to get the needed $\lambda$.

3.1.1. Potential Consensus under $\tilde{a}_{i j}=a_{i j}$. For $\tilde{a}_{i j}=a_{i j}$, we get $L\left(\widehat{n}_{\bar{\kappa}}\right)=L_{\widehat{n}_{\bar{\kappa}}}$, and the simplified protocol of (4) can be shown by

$$
u_{i}=\epsilon_{i} \sum_{j \in N_{i}(k)} a_{i j}\left[x_{j}(k)-x_{i}(k)\right] .
$$

Theorem 10. If $0 \leq b<1$ and $\epsilon_{i}$ satisfies

$$
0<\epsilon_{i}<\frac{1+b}{2 d_{i}}
$$

where $d_{i}=\sum_{j \in N_{i}} a_{j i}$, then under (15), each component of VSWSN (5) is the component potential consensus.

Proof. If $0 \leq b<1$, and $\epsilon_{i}$ satisfies (16), based on the Geřsgorin disc theorem, it holds that

$$
-1<b-2 d_{i} \epsilon_{i}<\lambda_{i}<b<1,
$$

hence $|\lambda|<1$. We have $\left[\left(B_{\widehat{n}}-\epsilon L_{\widehat{n}}\right)^{T}\right]^{m} L\left(\widehat{n}_{\bar{\kappa}}\right)\left(B_{\widehat{n}}-\epsilon L_{\widehat{n}}\right)^{m}<$ $\lambda^{2 m} \widetilde{d}_{i} I_{\widehat{n}}$. Following Theorem 7 , subsystem (5) is the potential consensus. This completes the proof.
Theorem 11. If $-1 \leq b<0$ and $\epsilon_{i}$ satisfies

$$
\frac{-1+b}{2 d_{i}}<\epsilon_{i}<0
$$

then under (15), subsystem (5) is the component potential consensus.

The proof is similar to that of Theorem 10, and it is omitted.

Remark 12. If $b=1$ and $V(\bar{\kappa})$ is the fixed node set, then the related consensus problems discussed here are the same as in literatures $[6,20]$. However, when $|b|>1$, the protocol (15) and the protocol listed in the literatures $[6,20]$ cannot insure subsystem (5) to achieve the consensus, and the following section will solve the problems.

In (15), if $\epsilon_{i}$ is equal to a certain value and $V(\bar{\kappa})$ is the fixed node set, then system converges to one equilibrium point. In order to get the wide consensus regions, the value of $\epsilon_{i}$ takes in $\left(0,(1+b) / 2 d_{i}\right)$, and the similarly reasons are taken by the Theorems 13 and 14 as follows.

3.1.2. Potential Consensus under $\tilde{a}_{i j}=b \xi / d_{i} \epsilon_{i}+a_{i j}$. When $|b| \geq 1$, for each node $i$, changes its weight appropriately, the related consensus problems can be transformed to the problems of $|b|<1$. If we choose $\tilde{a}_{i j}=b \xi / d_{i} \epsilon_{i}+a_{i j}$, then the protocol (4) can be expressed by

$$
u_{i}=\epsilon_{i} \sum_{j \in N_{i}(k)} a_{i j}\left[x_{j}(k)-x_{i}(k)\right]-b \xi x_{i}(k) .
$$

In this situation, the dynamic of each component in VSWSN can be shown by

$$
X_{\widehat{n}}(k+l)=\left(\widehat{B}_{\widehat{n}}-\epsilon L_{\widehat{n}}\right) X_{\widehat{n}}(k),
$$

where $\widehat{B}_{\widehat{n}}=\operatorname{diag}\{b(1-\xi), b(1-\xi), \ldots, b(1-\xi)\}, \epsilon=\operatorname{diag}\left\{\epsilon_{\widehat{1}}\right.$, $\left.\epsilon_{\widehat{2}}, \ldots, \epsilon_{\widehat{n}}\right\}$.

Based on Theorems 7 and 10, we get the following theorem.

Theorem 13. When $b \geq 1$, if $\epsilon_{i}$ and $\xi$ satisfy

$$
0<\epsilon_{i}<\frac{1+b(1-\xi)}{2 d_{i}}, \quad 1 \leq \xi<\frac{1}{b}+1,
$$

then, under (19), subsystem (20) is the component potential consensus.

Proof. According to Geřsgorin disc theorem, we obtain $\mid \lambda_{i}-$ $\epsilon_{i} d_{i}|\leq| \epsilon_{i} \mid d_{i}$. From (21), we get $-1<b(1-\xi)-2 \epsilon_{i} d_{i}<\lambda_{\bar{\kappa}}<$ $b(1-\xi)<1$. Similarly to the proof of Theorem 10, based on Theorem 7, it is known that subsystem (20) is the component potential consensus. This completes the proof.

Similarly to Theorem 13, we obtain theorem as follows.

Theorem 14. When $b \leq-1$, if $\epsilon_{i}$ and $\xi$ satisfy

$$
\frac{-1+b(1-\xi)}{2 d_{i}}<\epsilon_{i}<0, \quad 1<\xi<-\frac{1}{b}+1,
$$


then under protocol (19), subsystem (20) is the component potential consensus.

3.2. Consensus Investigation for WSN in the Varying Topology. Based on the discussion of the potential consensus, in this subsection, we investigate the consensus problems of the node attached component in VSWSN.

From analog equation (14), we get

$$
e_{\bar{\kappa}}\left(\widetilde{m}_{\kappa} l\right)=\Gamma X_{\widetilde{n}_{\bar{\kappa}}}\left(\widetilde{m}_{\kappa} l\right) .
$$

Let $\lambda_{\bar{\kappa}}=\max \left\{|\tilde{\lambda}| \mid \tilde{\lambda} \in \operatorname{eigen}\left(B_{\widehat{n}_{\bar{\kappa}}}-\epsilon_{\bar{n}_{\bar{\kappa}}} L_{\widehat{n}_{\bar{\kappa}}}\right)\right\}, y_{\bar{\kappa}}(k)=$ $\operatorname{norm}^{2}\left(X_{q_{\bar{\kappa}}^{\prime}}(k, \bar{\kappa})\right)$; it holds the following proposition.

Proposition 15. For all $C^{\prime}(\bar{\kappa}) \subset G(\bar{\kappa})$, where $C^{\prime}(\bar{\kappa})$ attaches on node $i_{\bar{\kappa}}$, it can be shown that

$$
\begin{aligned}
& \sum_{i_{\bar{\kappa}}, j_{\bar{\kappa}} \in V^{\prime}(\bar{\kappa})}\left\|\left[e_{\bar{\kappa}}^{i}\left(\widetilde{m}_{\kappa} l\right)-e_{\overline{\mathcal{\kappa}}}^{j}\left(\widetilde{m}_{\kappa} l\right)\right]\right\|^{2} \\
& \quad \leq \lambda_{\bar{\kappa}}^{2 m_{\kappa}} \cdots \lambda_{\overline{0}^{\prime}}^{2 m_{\overline{0}^{\prime}}} \tilde{d}_{i_{\bar{\kappa}}}\left\|X_{\overline{0}^{\prime}}(0)\right\|^{2}+\Lambda(\bar{\kappa}),
\end{aligned}
$$

where $\overline{0}^{\prime}, \overline{1}^{\prime}, \overline{2}^{\prime}, \ldots, \bar{\kappa}$ are the different topologies that $i_{\overline{\mathcal{\kappa}}}$ will appear successively as follows:

$$
\begin{gathered}
\Lambda(\bar{\kappa})=\widetilde{d}_{i_{\bar{\kappa}}}\left[\lambda_{\bar{\kappa}}^{2 m_{\kappa}} y_{\bar{\kappa}}\left(\widetilde{m}_{\kappa} l\right)+\cdots+\lambda_{\bar{\kappa}}^{2 m_{\kappa}} \cdots \lambda_{\overline{3}^{\prime}}^{2 m_{\overline{3}^{\prime}}} \lambda_{\overline{2}^{\prime}}^{2 m_{\overline{2}^{\prime}}} y_{\overline{2}^{\prime}}\left(\widetilde{m}_{2} l\right)\right. \\
\left.+\lambda_{\bar{\kappa}}^{2 m_{\kappa}} \cdots \lambda_{\overline{1}^{\prime}}^{2 m_{\overline{1}^{\prime}}} y_{\overline{1}^{\prime}}\left(\widetilde{m}_{1} l\right)\right] .
\end{gathered}
$$

Proof. Noting that

$$
\sum_{i_{\bar{\kappa}}, j_{\bar{\kappa}}}\left\|\left[e_{\bar{\kappa}}^{i}\left(\widetilde{m}_{\kappa} l\right)-e_{\bar{\kappa}}^{j}\left(\widetilde{m}_{\kappa} l\right)\right]\right\|^{2}=\left[e_{\bar{\kappa}}^{T}\left(\widetilde{m}_{\kappa} l\right) L\left(\widehat{n}_{\bar{\kappa}}\right) e_{\bar{\kappa}}\left(\widetilde{m}_{\kappa} l\right)\right],
$$

from (23), it results in

$$
\begin{aligned}
\sum_{i_{\bar{\kappa}}, j_{\bar{\kappa}}} \| & {\left[e_{\bar{\kappa}}^{i}\left(\widetilde{m}_{\kappa} l\right)-e_{\bar{\kappa}}^{j}\left(\widetilde{m}_{\kappa} l\right)\right] \|^{2} } \\
= & {\left[X_{\widehat{n}_{\bar{\kappa}}}\left(\widetilde{m}_{\kappa} l\right)^{T} \Gamma^{T} L\left(\widehat{n}_{\bar{\kappa}}\right) \Gamma X_{\widehat{n}_{\bar{\kappa}}}\left(\widetilde{m}_{\kappa} l\right)\right] } \\
= & {\left[X_{\widehat{n}_{\bar{\kappa}}}\left(\left(\widetilde{m}_{\kappa-1}+1\right) l\right)^{T}\left(B_{\widehat{n}_{\bar{\kappa}}}-\epsilon_{\bar{\kappa}} L_{\widehat{n}_{\bar{\kappa}}}\right)^{m_{\kappa}} \Gamma^{T} L\left(\widehat{n}_{\bar{\kappa}}\right) \Gamma\right.} \\
& \left.\times\left(B_{\widehat{n}_{\bar{\kappa}}}-\epsilon_{\bar{\kappa}} L_{\widehat{n}_{\bar{\kappa}}}\right)^{m_{\kappa}} X_{\widehat{n}_{\bar{\kappa}}}\left(\left(\widetilde{m}_{\kappa-1}+1\right) l\right)\right] .
\end{aligned}
$$

Since the absolute of each eigenvalue of $\Gamma$ is no more than 1 , the absolute of each eigenvalue of $L\left(\widehat{n}_{\bar{\kappa}}\right)$ is no more than $\widetilde{d}_{i_{\bar{\kappa}}}$, and the absolute of each eigenvalue of $B_{\widehat{n}_{\bar{\kappa}}}-\epsilon L_{\widehat{n}_{\bar{\kappa}}}$ is no more than $\lambda_{\bar{\kappa}}$. The following inequality is obtained:

$$
\begin{aligned}
& {\left[e_{\bar{\kappa}}^{T}\left(\widetilde{m}_{\kappa} l\right) L\left(\widehat{n}_{\bar{\kappa}}\right) e_{\bar{\kappa}}\left(\widetilde{m}_{\kappa} l\right)\right]} \\
& \quad \leq \lambda_{\overline{\bar{\kappa}}}^{2 m_{\kappa}} \widetilde{d}_{i_{\bar{\kappa}}} X_{\widehat{n}_{\bar{\kappa}}}\left(\left(\widetilde{m}_{\kappa-1}+1\right) l\right)^{T} X_{\widehat{n}_{\bar{\kappa}}}\left(\left(\widetilde{m}_{\kappa-1}+1\right) l\right) .
\end{aligned}
$$

On the other hand,

$$
\begin{aligned}
X_{\widehat{n}_{\bar{\kappa}}} & \left(\left(\widetilde{m}_{\kappa-1}+1\right) l\right)^{T} X_{\widehat{n}_{\bar{\kappa}}}\left(\left(\widetilde{m}_{\kappa-1}+1\right) l\right) \\
\leq & X_{p_{\bar{\kappa}}}\left(\left(\widetilde{m}_{\kappa-1}+1\right) l\right)^{T} X_{p_{\bar{\kappa}}}\left(\left(\widetilde{m}_{\kappa-1}+1\right) l\right) \\
\quad & \quad X_{q_{\bar{\kappa}}^{\prime}}\left(\left(\widetilde{m}_{\kappa-1}+1\right) l\right)^{T} X_{q_{\bar{\kappa}}^{\prime}}\left(\left(\widetilde{m}_{\kappa-1}+1\right) l\right) \\
\leq & X_{p_{\bar{\kappa}}}\left(\left(\widetilde{m}_{\kappa-1}+1\right) l\right)^{T} X_{p_{\bar{\kappa}}}\left(\left(\widetilde{m}_{\kappa-1}+1\right) l\right)+y_{\bar{\kappa}}\left(\widetilde{m}_{\kappa-1}\right) l,
\end{aligned}
$$

where $X_{q_{\bar{\kappa}}^{\prime}}(k)$ refers to the state vector of all newly jointed nodes in component $C^{\prime}(\bar{\kappa}) ; X_{p_{\bar{\kappa}}}(k)$ means the state vector of the component which attaches on node $i_{\bar{\kappa}}$ and all related valid nodes that come from the former topology $\overline{\kappa-1}$.

Considering some nodes in topology $\overline{\kappa-1}$ may be invalid at time $\widetilde{m}_{\kappa} l$, the node attached component of $i_{\bar{\kappa}}$ in the topology $\overline{\kappa-1}$ contains $1_{\bar{\kappa}}, \ldots, p_{\bar{\kappa}}$ and may contain other nodes. In addition, the values of $1_{\bar{\kappa}}, \ldots, p_{\bar{\kappa}}$ at time $k$ are equal to the $1_{\overline{\kappa-1}}, \ldots, p_{\overline{\kappa-1}}$ at time $k-l$, then it holds

$$
X_{p_{\bar{\kappa}}}(k)^{T} X_{p_{\bar{\kappa}}}(k) \leq X_{\widehat{n}_{\overline{k-1}}}(k-l)^{T} X_{\widehat{n}_{\overline{k-1}}}(k-l) .
$$

Following (29), (30), it can be shown that

$$
\begin{aligned}
{\left[X_{\widehat{n}_{\bar{\kappa}}}\left(\widetilde{m}_{\kappa} l\right)^{T} L\left(\widehat{n}_{\bar{\kappa}}\right) X_{\widehat{n}_{\bar{\kappa}}}\left(\widetilde{m}_{\kappa} l\right)\right] } \\
\leq \lambda_{\bar{\kappa}}^{2 m_{\kappa}} \widetilde{d}_{i_{\bar{\kappa}}}\left[X_{\widehat{n}_{\bar{\kappa}-1}}\left(\left(\widetilde{m}_{\kappa-1}+1\right) l\right)^{T} X_{\widehat{n}_{\bar{\kappa}-1}}\left(\left(\widetilde{m}_{\kappa-1}+1\right) l\right)\right. \\
\left.\quad+y_{\bar{\kappa}}\left(\widetilde{m}_{\kappa-1}\right) l\right] .
\end{aligned}
$$

Similaring to (31), we have

$$
\begin{gathered}
X_{\widehat{n}_{\overline{\kappa-1}}}\left(\left(\widetilde{m}_{\kappa-1}+1\right) l\right)^{T} X_{\widetilde{n}_{\overline{\kappa-1}}}\left(\left(\widetilde{m}_{\kappa-1}+1\right) l\right) \\
\leq \lambda_{\widetilde{\kappa-1}^{2\left(m_{\kappa-1}\right)}}\left[X_{p_{\overline{\kappa-1}}}\left(\left(\widetilde{m}_{\kappa-2}+1\right) l\right)^{T} X_{p_{\overline{\kappa-1}}}\left(\left(\widetilde{m}_{\kappa-2}+1\right) l\right)\right. \\
\left.+y_{\overline{\kappa-1}}\left(\widetilde{m}_{\kappa-2}\right) l\right] .
\end{gathered}
$$

Combining (32) and (31), we get

$$
\begin{aligned}
& {\left[X_{\bar{\kappa}}\left(\widetilde{m}_{\kappa} l\right)^{T} L\left(\widehat{n}_{\bar{\kappa}}\right) X_{\widehat{n}_{\bar{\kappa}}}\left(\widetilde{m}_{\kappa} l\right)\right]} \\
& \leq \widetilde{d}_{i_{\bar{\kappa}}}\left[\lambda_{\bar{\kappa}}^{2 m_{\kappa}} \lambda_{\overline{\kappa-1}}^{2 m_{\kappa-1}} X_{p_{\overline{\kappa-1}}}\left(\left(\widetilde{m}_{\kappa-2}+1\right) l\right)^{T} X_{p_{\overline{\kappa-1}}}\left(\left(\widetilde{m}_{\kappa-2}+1\right) l\right)\right. \\
& \left.+\lambda_{\bar{\kappa}}^{2 m_{\kappa}} \lambda_{\overline{\kappa-1}}^{2 m_{\kappa-1}} y_{\overline{\kappa-1}}\left(m_{\kappa-1} l\right)+\lambda_{\bar{\kappa}}^{2 m_{\kappa}} y_{\bar{\kappa}}\left(m_{\kappa} l\right)\right] .
\end{aligned}
$$

Based on (30), (31), (32), and (33), by the limited iterative, we get (24).

Theorem 16. If each component (5) of WSN is LLI connected, then the following statements hold. (i) If $0 \leq b<1, \epsilon_{i_{\bar{\kappa}}}$ satisfies (16), and (34) holds, then under protocol (15), each component (5) achieves the component consensus. 
(ii) If $-1<b \leq 0, \epsilon_{i_{\bar{\kappa}}}$ satisfies (18), and (34) holds, then under protocol (15), each component (5) achieves the component consensus, where

$$
\lim _{m \rightarrow \infty} \Lambda(\bar{\kappa})=0
$$

In addition, if subsystem (5) is GLI connected, then under protocol (15), subsystem (5) achieves the global consensus.

Proof. (i) When $0 \leq b<1$ and $\epsilon_{i_{\bar{\kappa}}}$ satisfies (16), if WSN is LLI connected and (34) holds, then for all $i_{\kappa^{\prime}}, j_{\mathcal{K}^{\prime}} \in V\left(\kappa^{\prime}\right)$, there exist two related node attached components $C^{\prime}$ and $C^{\prime \prime}$ of $i_{\kappa^{\prime}}$ and $j_{\kappa^{\prime}}$, respectively, such that in the jointed topology $\kappa_{0},\left(\kappa^{\prime} \leq \kappa_{0}\right)$ Proposition 15 holds.

Based on Theorem 10, Assumption 1, subsystem (5) is the component potential consensus, then

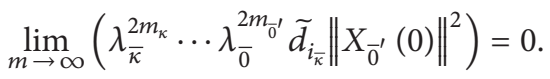

On the other hand, if (34) holds, then $\lim _{m \rightarrow \infty}\left(\sum_{i_{\bar{\kappa}}, j_{\bar{\kappa}} \in V^{\prime}(\bar{\kappa})}\left\|\left[e_{\bar{\kappa}}^{i}\left(\widetilde{m}_{\kappa} l\right)-e_{\bar{\kappa}}^{j}\left(\widetilde{m}_{\kappa} l\right)\right]\right\|^{2}\right)=0$.

So it holds $\lim _{m \rightarrow \infty} x_{i}(k, \bar{\kappa})=x_{j}(k, \bar{\kappa})$.

(ii) Similarly, we can get the result when $-1<b \leq 0$; we also can show that VSWSN is the global consensus when WSN is GLI connected. This completes the proof.

Theorem 17. If component (5) is LLI connected, then the following statements hold. (i) If $b \geq 1, \epsilon_{i_{\bar{\Gamma}}}, \xi$ satisfy (21), and if (34) holds, then under (19), subsystem (20) is the component consensus.

(ii) If $-1 \geq b, \epsilon_{i_{\vec{k}}}, \xi$ satisfy (22), and if (34) holds, then under (19), subsystem (20) is the component consensus.

In addition, if VSWSN (5) is GLI connected, then under protocol (19), VSWSN (20) is the global consensus.

Proof. Similar to the proof of Theorem 16, it is omitted.

\section{The Consensus Regions}

In fact, the former sections discuss consensus problems that require the state of each node keeps in coordination with the average value $x^{0}(k, \bar{\kappa})$, and the value of $x^{0}(k, \bar{\kappa})$ is decided by the parameters $\epsilon_{i_{\bar{\kappa}}}$ and $\xi$. The regions of $\epsilon_{i_{\bar{\kappa}}}$ and $\xi$ mean that the changeable value of $x^{0}(k, \bar{\kappa})$ has a widely range. Hence, in this section, we investigate the consensus regions.

Definition 18. Under protocol (4) or (19), the region of $\epsilon_{i_{\bar{\kappa}}}$ and $\xi$, such that VSWSN (5) is the component consensus or the global consensus, is called the consensus region.

In the varying topology, denote $d_{i_{\bar{\kappa}}}=\sum_{j_{\bar{\kappa}} \in N i_{\bar{\kappa}}} a_{i j}(\overline{\mathcal{K}})$,

$$
\begin{aligned}
& \Psi_{i_{\bar{\kappa}}}^{1}=\left\{\frac{-1+b}{2 d_{i_{\bar{\kappa}}}}<\epsilon_{i_{\bar{\kappa}}}<0\right\}, \\
& \Psi_{i_{\bar{\kappa}}}^{2}=\left\{0<\epsilon_{i_{\bar{\kappa}}}<\frac{1+b}{2 d_{i_{\bar{\kappa}}}}\right\},
\end{aligned}
$$

$$
\begin{gathered}
\Upsilon_{i_{\bar{\kappa}}}^{1}=\left\{\frac{-1+b(1-\xi)}{2 d_{i_{\bar{\kappa}}}}<\epsilon_{i_{\bar{\kappa}}}<0\right\}, \\
\Upsilon_{i_{\bar{\kappa}}}^{2}=\left\{0<\epsilon_{i_{\bar{\kappa}}}<\frac{1+b(1-\xi)}{2 d_{i_{\bar{\kappa}}}}\right\}, \\
\Xi_{\bar{\kappa}}^{1}=\left\{1<\xi<-\frac{1}{b}+1\right\}, \quad \Xi_{\bar{\kappa}}^{2}=\left\{1<\xi<\frac{1}{b}+1\right\}, \\
\Psi_{\bar{\kappa}}^{1}=\prod_{i \in V^{\prime}(\bar{\kappa})} \Psi_{i_{\bar{\kappa}}}^{1}, \quad \Psi_{\bar{\kappa}}^{2}=\prod_{i \in V^{\prime}(\bar{\kappa})} \Psi_{i_{\bar{\kappa}}}^{2}, \\
\Upsilon_{\bar{\kappa}}^{1}=\prod_{i \in V^{\prime}(\bar{\kappa})} \Upsilon_{i_{\bar{\kappa}}}^{1}, \quad \Upsilon_{\bar{\kappa}}^{2}=\prod_{i \in V^{\prime}(\bar{\kappa})} \Upsilon_{i_{\bar{\kappa}}}^{2},
\end{gathered}
$$

and following Theorems 7, 10, and 13, we get the following corollary.

Corollary 19. Under protocol (4) or (19), if $G(\bar{\kappa})$ is $L L I$ connected or GLI connected, then the following statements hold.

(1) If $0 \leq b<1$, then for all $\epsilon_{\bar{\kappa}} \in \Psi_{\bar{\kappa}}^{2}$, system (5) achieves the component potential consensus or the global potential consensus, respectively.

(2) If $-1<b<0$, then for all $\epsilon_{\bar{\kappa}} \in \Psi_{\bar{\kappa}}^{1}$, system (5) achieves the component potential consensus or the global potential consensus, respectively.

(3) If $b \geq 1$, then for all $\epsilon_{\bar{\kappa}} \times \xi \in \Upsilon_{\bar{\kappa}}^{2} \times \Xi_{\overline{\mathcal{K}}}^{2}$, system (20) achieves the component potential consensus or the global potential consensus, respectively.

(4) If $b \leq-1$, then for all $\epsilon_{\bar{\kappa}} \times \xi \in \Upsilon_{\bar{\kappa}}^{1} \times \Xi_{\bar{\kappa}}^{1}$, system (20) achieves the component potential consensus or the global potential consensus, respectively.

Following Theorem 16 and Corollary 19, it holds the following results.

Corollary 20. Under protocol (4) or (19), if $G(\bar{\kappa})$ is $L L I$ connected or GLI connected and (34) holds, then the following statements hold.

(1) If $0 \leq b<1$, then for all $\epsilon_{\bar{\kappa}} \in \Psi_{\bar{\kappa}}^{2}$, system (5) achieves the component consensus or the global consensus, respectively.

(2) If $-1<b<0$, then for all $\epsilon_{\bar{\kappa}} \in \Psi_{\overline{\mathcal{K}}}^{1}$, system (5) achieves the component consensus or the global consensus, respectively.

(3) If $b \geq 1$, then for all $\epsilon_{\bar{\kappa}} \times \xi \in \Upsilon_{\bar{\kappa}}^{2} \times \Xi_{\bar{\kappa}}^{2}$, system (20) achieves the component consensus or the global consensus, respectively.

(4) If $b \leq-1$, then for all $\epsilon_{\overline{\mathcal{\kappa}}} \times \xi \in \Upsilon_{\bar{\kappa}}^{1} \times \Xi_{\overline{\mathcal{\kappa}}}^{1}$, system (20) achieves the component consensus or the global consensus, respectively.

Proof. (1) If $G(\bar{\kappa})$ is LLI connected, based on Corollary 19, when $0 \leq b<1$, for all $\epsilon_{\bar{\kappa}} \in \Psi_{i_{\bar{\kappa}}}^{2}$, under (4), WSN is 
the potential consensus. Following Theorem 16, if (34) holds, then subsystem (5) is the component consensus. Analogously, we can proof (2), (3), and (4).

\section{Numerical Example}

Example 21. Consider two node attached components $C^{\prime}(\bar{\kappa})$ and $C^{\prime \prime}(\bar{\kappa})$ of $1_{\bar{\kappa}}, 3_{\bar{\kappa}}$ (as shown in Figure 1 ), where $\overline{0}, \overline{1}$, and $\overline{2}$ are the part topologies in sequence, $a_{i j}(\bar{\kappa})$ takes value in 0 or 1 , and $C^{\prime}(\overline{1})=C^{\prime \prime}(\overline{1})$ is the intersection topology. For $C^{\prime}(\overline{\mathcal{\kappa}})$ and $C^{\prime \prime}(\bar{\kappa})$, the related node set sequences are shown as follows:

$$
\begin{gathered}
V^{\prime}(\overline{0})=\left\{1 \overline{1}_{\overline{0}}, 2_{\overline{0}}, 6_{\overline{0}}\right\}, \quad V^{\prime}(\overline{1})=\left\{1_{\overline{1}}, 2_{\overline{1}}, 3_{\overline{1}}, 4_{\overline{1}}, 5_{\overline{1}}\right\}, \\
V^{\prime}(\overline{2})=\left\{1_{\overline{2}}, 2_{\overline{2}}\right\}, \ldots, \quad V^{\prime \prime}(\overline{0})=\left\{3_{\overline{0}}, 4_{\overline{0}}\right\}, \\
V^{\prime \prime}(\overline{1})=\left\{1_{\overline{1}}, 2_{\overline{1}}, 3_{\overline{1}}, 4_{\overline{1}}, 5_{\overline{1}}\right\}, \quad V^{\prime \prime}(\overline{2})=\left\{3_{\overline{2}}, 5_{\overline{2}}\right\}, \ldots .
\end{gathered}
$$

Suppose that the state of $i_{\bar{\kappa}}$ is shown as in (3), and consider the following three questions:

(i) when $b=0.6$ or $b=-0.6$, in topology $\overline{0}$, under protocol (15), determine whether system (5) achieves the component potential consensus or not;

(ii) when $b=5$ or $b=-5$, in topology $\overline{0}$, under protocol (19), determine whether system (20) achieves the component potential consensus or not;

(iii) suppose that WSN is LLI connected, the partial node attached components of 1,3 are in topology sequence $\overline{0}, \overline{1}$, and $\overline{2}$ (as shown in Figure 1 ), and $b=0.6$, under protocol (15), determine whether system (5) achieves the component consensus or not. When WSN is GLI connected, two node attached components of 1,3 are in topology sequence $\overline{0}, \overline{1}$ (as shown in Figure 1 ), under protocol (15), determine whether system (5) achieves the global consensus or not.

For the above questions, under the related protocol, we can get (34) holds firstly. Then we propose the following analysis.

For the first question, when $b=0.6$, from (16), we get the consensus region of $\overline{0}$ as follows:

$$
\begin{aligned}
& \Psi_{\overline{0}}^{2}=\prod_{i \in V^{\prime}(\overline{0})} \Psi_{\overline{\overline{0}}_{\overline{0}}}^{2}, \\
& \Psi_{i_{\overline{0}}}^{2}=\left\{\epsilon_{i_{\overline{0}}} \mid 0<\epsilon_{i_{\overline{0}}}<\frac{0.8}{d_{i_{\overline{0}}}}\right\},
\end{aligned}
$$

$i_{\overline{0}} \in\{1,2,3,4,6\}$.

Choosing $(0.5,0.3,0.5,0.7,0.6)^{T} \in \Psi_{\overline{0}}^{2}$, we get $\epsilon_{\overline{0}}=\operatorname{diag}\{0.5$, $0.3,0.5,0.7,0.6\}$; when $l=0.2, m_{0}=10$, the simulation result is shown in Figure 2.
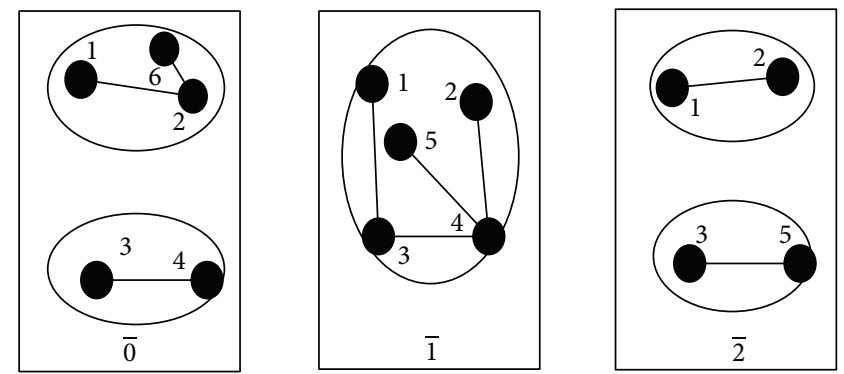

Figure 1: For nodes 1 and 3, there are two related node attached components.

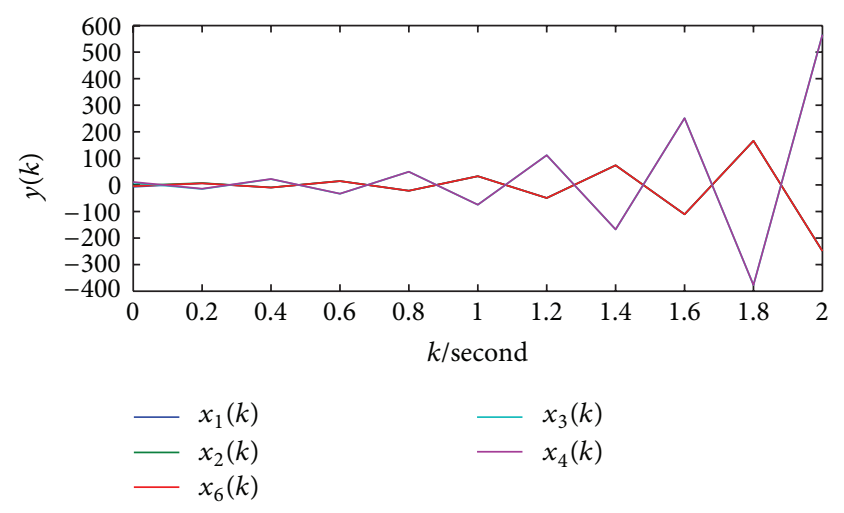

FIGURE 2: In topology $\overline{0}$, the node attached components of 1 and 3 achieve the potential consensus, respectively.

When $b=-0.6$, from (16), we get the consensus region of topology $\overline{0}$ as follows:

$$
\begin{aligned}
& \Psi_{\overline{0}}^{1}=\prod_{i \in V^{\prime}(\overline{0})} \Psi_{i_{\overline{0}}}^{1}, \\
& \Psi_{i_{\overline{0}}}^{1}=\left\{\epsilon_{i_{\overline{0}}} \mid \frac{-0.8}{d_{i_{\overline{0}}}}<\epsilon_{i_{\overline{0}}}<0\right\}, \\
& i_{\overline{0}} \in\{1,2,3,4,6\} .
\end{aligned}
$$

Choosing $(-0.55,-0.3,-0.5,-0.6,-0.4)^{T} \in \Psi_{\overline{0}}^{1}$, we get $\epsilon_{\overline{0}}=$ $\operatorname{diag}\{-0.55,-0.3,-0.5,-0.6,-0.4\}$; when $l=0.2, m_{0}=10$, the simulation result is shown in Figure 3.

For the second question, when $b=5$, from (21), we get the consensus region $Y_{\overline{0}}^{2}=\prod_{i_{\overline{0}} \in V^{\prime}(\overline{0})} Y_{i_{\overline{0}}}^{2}$, where

$$
\begin{aligned}
& \Upsilon_{\overline{0}}^{2}=\left\{0<\epsilon_{i_{\overline{0}}}<\frac{1+5(1-\xi)}{2 d_{i}}\right\}, \\
& \Xi_{\overline{0}}^{2}=\left\{1<\xi<\frac{6}{5}\right\}, \\
& i_{\overline{0}} \in\{1,2,3,4,6\} .
\end{aligned}
$$

Choosing $0.9 \times(0.5,0.3,0.5,0.7,0.6)^{T} \in \Xi^{2} \times \Upsilon_{\overline{0}}^{2}$, we get $\epsilon_{\overline{0}}=\operatorname{diag}\{0.5,0.3,0.6,0.7,0.65\}$; when $l=0.2, m_{0}=10$, the simulation result is shown as Figure 4. 


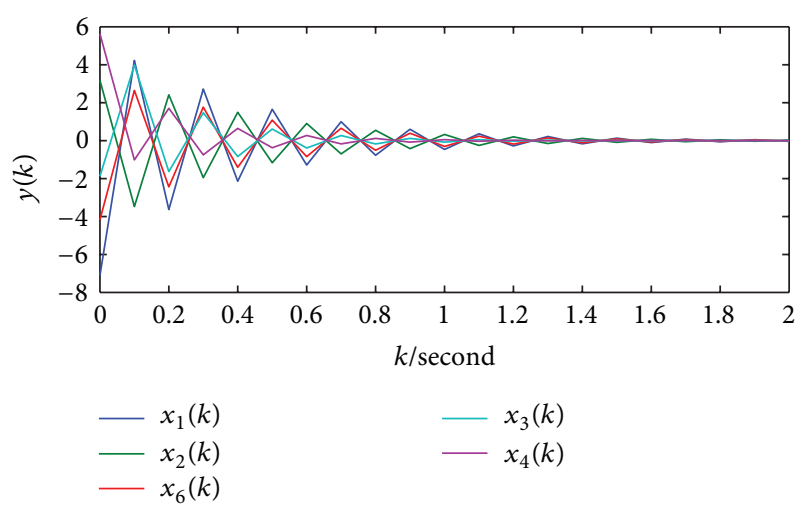

Figure 3: In topology $\overline{0}$, node attached components of 1 and 3 keep the component potential consensus, respectively.

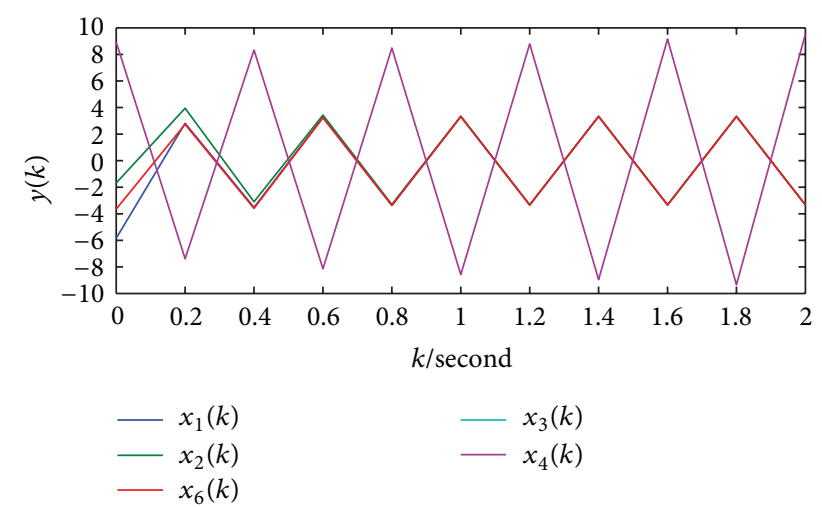

FIGURE 4: In topology $\overline{0}$, node attached components of 1 and 3 keep the component potential consensus, respectively.

When $b=-5$, from (21), we get the consensus region $\Upsilon_{\overline{0}}^{1}=$ $\prod_{i_{\overline{0}} \in V^{\prime}(\overline{0})} Y_{i_{\overline{0}}}^{1}$, where

$$
\begin{aligned}
& \Upsilon_{i_{\overline{0}}}^{1}=\left\{\frac{-1+5(1-\xi)}{2 d_{i_{\overline{0}}}}<\epsilon_{i_{\overline{0}}}<0\right\}, \\
& \Xi_{\overline{0}}^{1}=\left\{1<\xi<\frac{6}{5}\right\}, \\
& i_{\overline{0}} \in\{1,2,3,4,6\} .
\end{aligned}
$$

Choosing $0.9 \times(0.5,0.3,0.5,0.7,0.6)^{T} \in \Xi^{1} \times \Upsilon_{\frac{1}{0}}^{1}$, we have $\epsilon_{\overline{0}}=\operatorname{diag}\{-0.6,-0.2,-0.5,-0.5,-0.3\}$; when $l=0.2, m_{0}=10$, the simulation result is shown in Figure 5.

For the last question, when $b=0.6$, from (21) we get the consensus region of $\bar{\kappa}$ below, where $\bar{\kappa} \in\{\overline{0}, \overline{1}, \overline{2}\}$,

$$
\Psi_{\bar{\kappa}}^{2}=\prod_{i \in V^{\prime}(\bar{\kappa})} \Psi_{i_{\bar{\kappa}}}^{2}, \quad \Psi_{i_{\bar{\kappa}}}^{2}=\left\{0<\epsilon_{i_{\bar{\kappa}}}<\frac{1+5(1-\xi)}{2 d_{i_{\bar{\kappa}}}}\right\} .
$$

Similarly, we choose $\epsilon_{\overline{0}}=\operatorname{diag}\{0.5,0.3,0.6,0.7,0.65\}, \epsilon_{i_{\overline{1}}}=$ $\epsilon_{i_{\overline{2}}}=\operatorname{diag}\{0.5,0.3,0.6,0.7,0.65\}$. When $l=0.2$, if VSWSN is LLI connected, the part simulation results are shown in Figures 2, 6, and 7. And if VSWSN is GLI connected, the simulation results are shown in Figures 2 and 6.

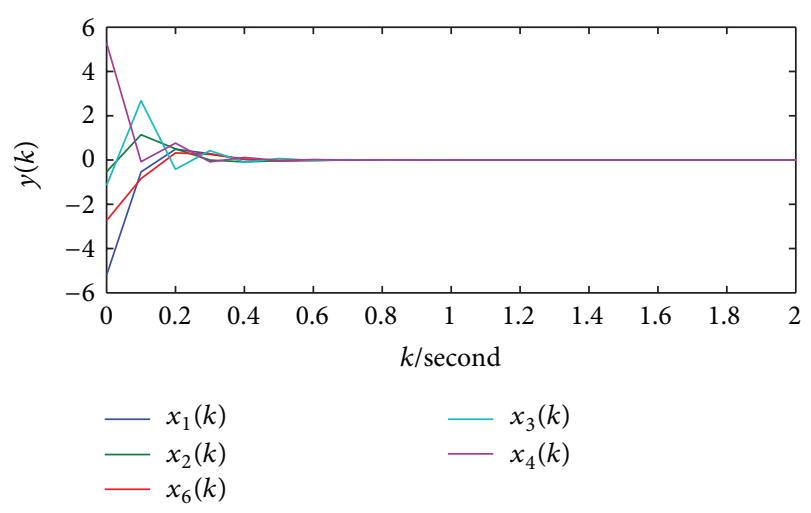

FIGURE 5: In topology $\overline{0}$, node attached components of 1 and 3 keep the component potential consensus, respectively.

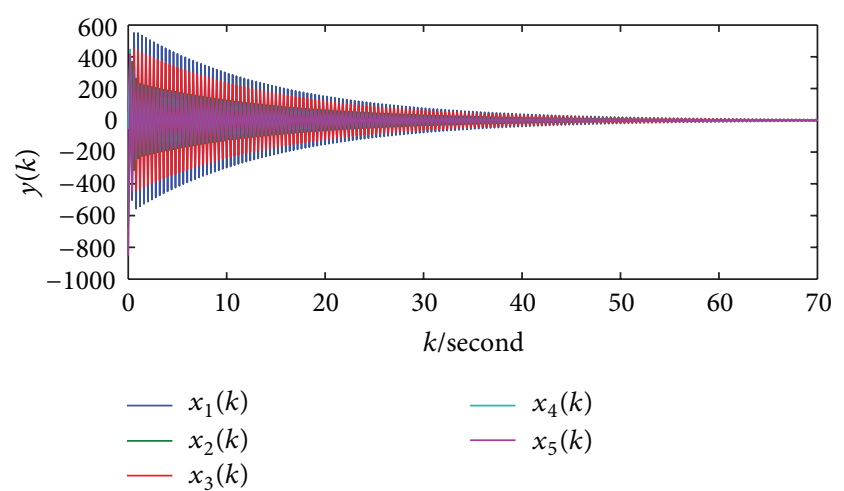

FIGURE 6: In topology $\overline{1}$, node attached components of 1 and 3 keep the component potential consensus, respectively.

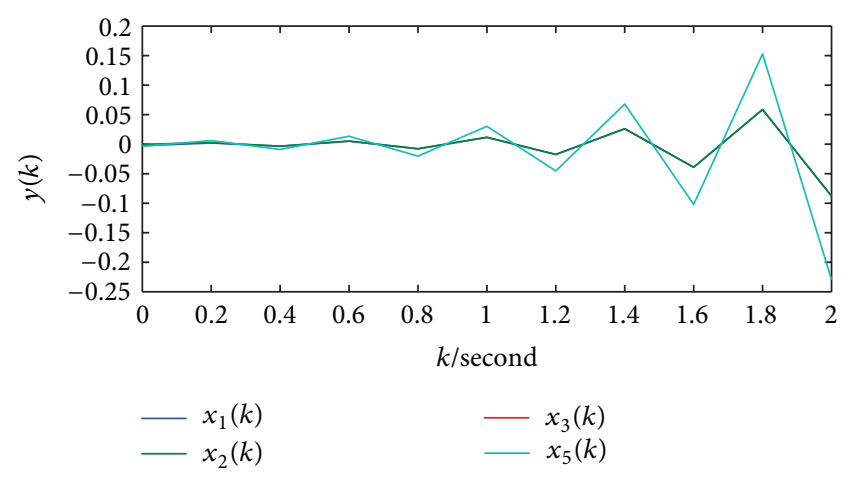

FIGURE 7: In topology $\overline{2}$, node attached components of 1 and 3 keep the component potential consensus, respectively.

\section{Conclusions}

This paper has investigated the consensus problems of VSWSN. According to the local limited intersection connected and the global limited intersection connected, it has provided several criterions of the component consensus and the global consensus. All gotten results have been based on the structures of the node attached component sequences. Based on the structure, the results cannot be influenced by 
the increasing-decreasing node set. In the meanwhile, it has given the consensus regions.

\section{Acknowledgments}

This work is supported by the National Natural Science Foundation of China (61075060), the innovation program of Shanghai Municipal Commission (12zz064), and the Open Project of State Key Laboratory of Industrial Control Technology (ICT1231).

\section{References}

[1] I. F. Akyildiz, W. Su, Y. Sankarasubramaniam, and E. Cayirci, "A survey on sensor networks," IEEE Communications Magazine, vol. 40, no. 8, pp. 102-114, 2002.

[2] Y. C. Wu, Q. Chaudhari, and E. Serpedin, "Clock synchronization of wireless sensor networks," IEEE Signal Processing Magazine, vol. 28, no. 1, pp. 124-138, 2011.

[3] H. Luo, H. Tao, H. Ma, and S. K. Das, "Data fusion with desired reliability in wireless sensor networks," IEEE Transactions on Parallel and Distributed Systems, vol. 23, no. 3, pp. 501-513, 2011.

[4] Y. Xia, J. Shang, J. Chen, and G. P. Liu, "Networked data fusion with packet losses and variable delays," IEEE Transactions on Systems, Man, and Cybernetics B, vol. 39, no. 5, pp. 1107-1120, 2009.

[5] M. Defoort, T. Floquet, A. Kökösy, and W. Perruquetti, "Slidingmode formation control for cooperative autonomous mobile robots," IEEE Transactions on Industrial Electronics, vol. 55, no. 11, pp. 3944-3953, 2008.

[6] R. Olfati-Saber and R. M. Murray, "Consensus problems in networks of agents with switching topology and time-delays," IEEE Transactions on Automatic Control, vol. 49, no. 9, pp. 15201533, 2004.

[7] W. Yu, G. Chen, and M. Cao, "Some necessary and sufficient conditions for second-order consensus in multi-agent dynamical systems," Automatica, vol. 46, no. 6, pp. 1089-1095, 2010.

[8] W. Yu, G. Chen, and J. Lü, "On pinning synchronization of complex dynamical networks," Automatica, vol. 45, no. 2, pp. 429-435, 2009.

[9] M. Zhu and S. Martínez, "Discrete-time dynamic average consensus," Automatica, vol. 46, no. 2, pp. 322-329, 2010.

[10] Y. G. Sun, L. Wang, and G. Xie, "Average consensus in networks of dynamic agents with switching topologies and multiple timevarying delays," Systems \& Control Letters, vol. 57, no. 2, pp. 175183, 2008.

[11] P. Lin and Y. Jia, "Average consensus in networks of multi-agents with both switching topology and coupling time-delay," Physica A, vol. 387, no. 1, pp. 303-313, 2008.

[12] H. Atrianfar and M. Haeri, "Average consensus in networks of dynamic multi-agents with switching topology: infinite matrix products," ISA Transctions, vol. 51, pp. 522-530, 2012.

[13] W. Zhou, J. Mou, T. Wang, C. Ji, and J. Fang, "Targetsynchronization of the distributed wireless sensor networks under the same sleeping-awaking method," Journal of the Franklin Institute, vol. 349, no. 6, pp. 2004-2018, 2012.

[14] W. Zhou, J. Mou, T. Wang, C. Ji, and J. Fang, "Quasi-average mean square consensus for wireless sensor networks under three topologies with respect to sleeping-awaking method," Optimal Control Applications and Methods, 2012.
[15] H. Wang and Y. Guo, "Consensus on scale-free network," in Proceedings of the Amercia Control Conference, pp. 748-752, Seattle, Wash, USA, June 2008.

[16] H. Yang, L. Lan, and S. Zhang, "Consensus of synchronizationpreferential scale free netwoeks," Physics Procedia, vol. 3, pp. 1913-1920, 2010.

[17] Z. Li, Z. Duan, G. Chen, and L. Huang, "Consensus of multiagent systems and synchronization of complex networks: a unified viewpoint," IEEE Transactions on Circuits and Systems. I, vol. 57, no. 1, pp. 213-224, 2010.

[18] W. Yu, G. Chen, W. Ren, J. Kurths, and W. X. Zheng, "Distributed higher order consensus protocols in multiagent dynamical systems," IEEE Transactions on Circuits and Systems. I, vol. 58, no. 8, pp. 1924-1932, 2011.

[19] C. Liu, Z. Duan, G. Chen, and L. Huang, "Analyzing and controlling the network synchronization regions," Physica A, vol. 386, no. 1, pp. 531-542, 2007.

[20] T. Li, M. Fu, L. Xie, and J.-F. Zhang, "Distributed consensus with limited communication data rate," IEEE Transactions on Automatic Control, vol. 56, no. 2, pp. 279-292, 2011. 


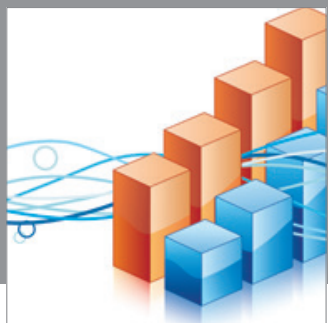

Advances in

Operations Research

mansans

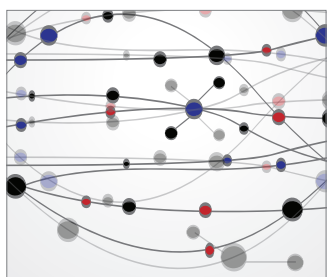

The Scientific World Journal
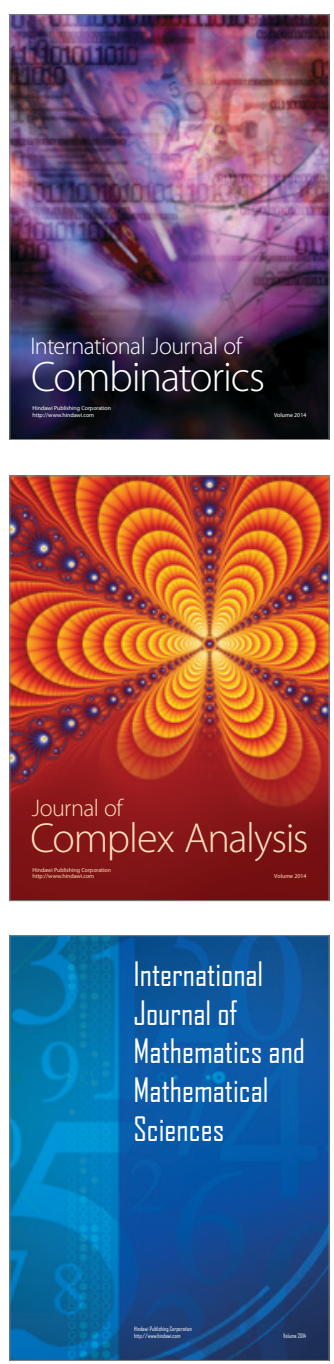
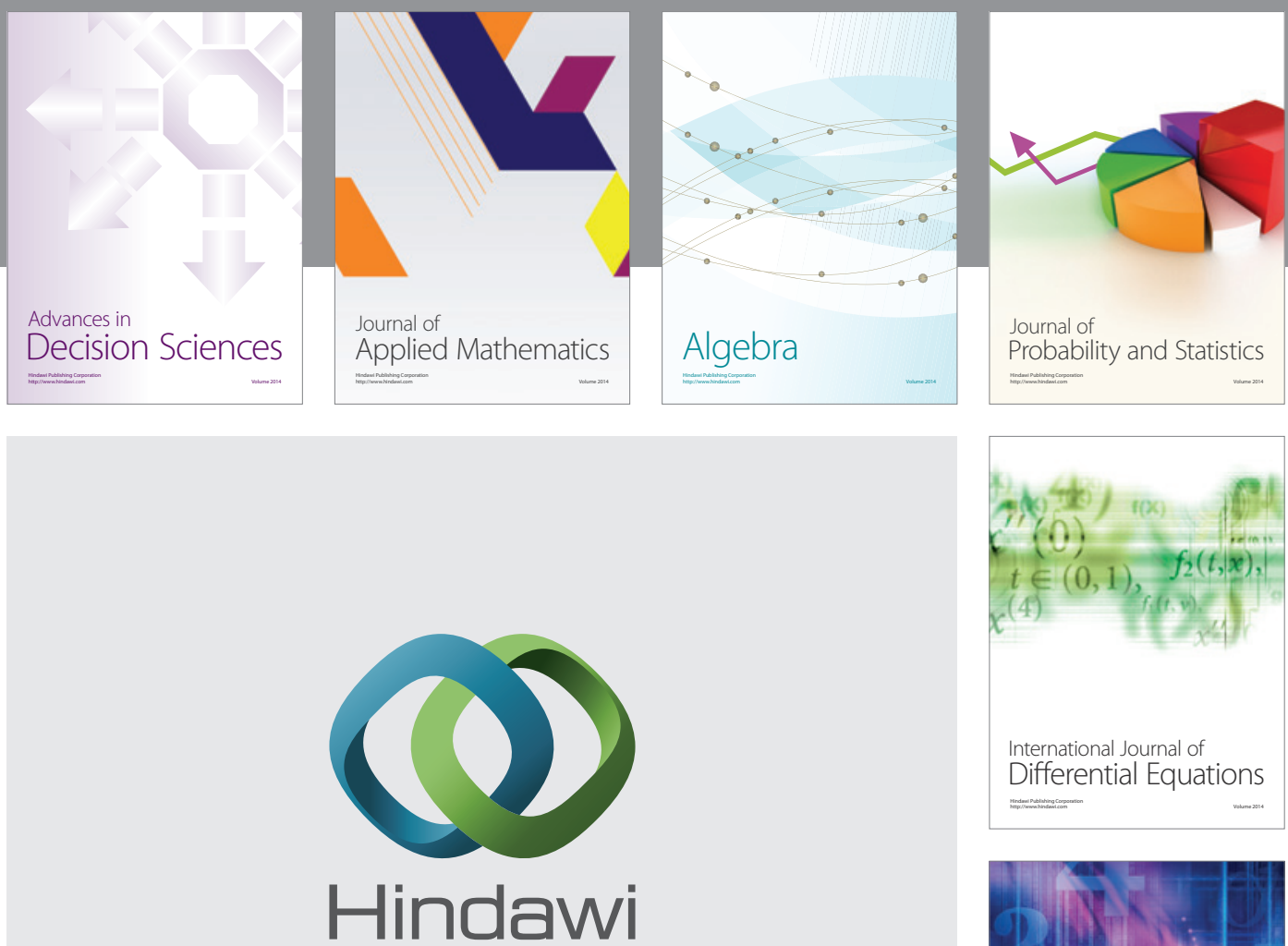

Submit your manuscripts at http://www.hindawi.com
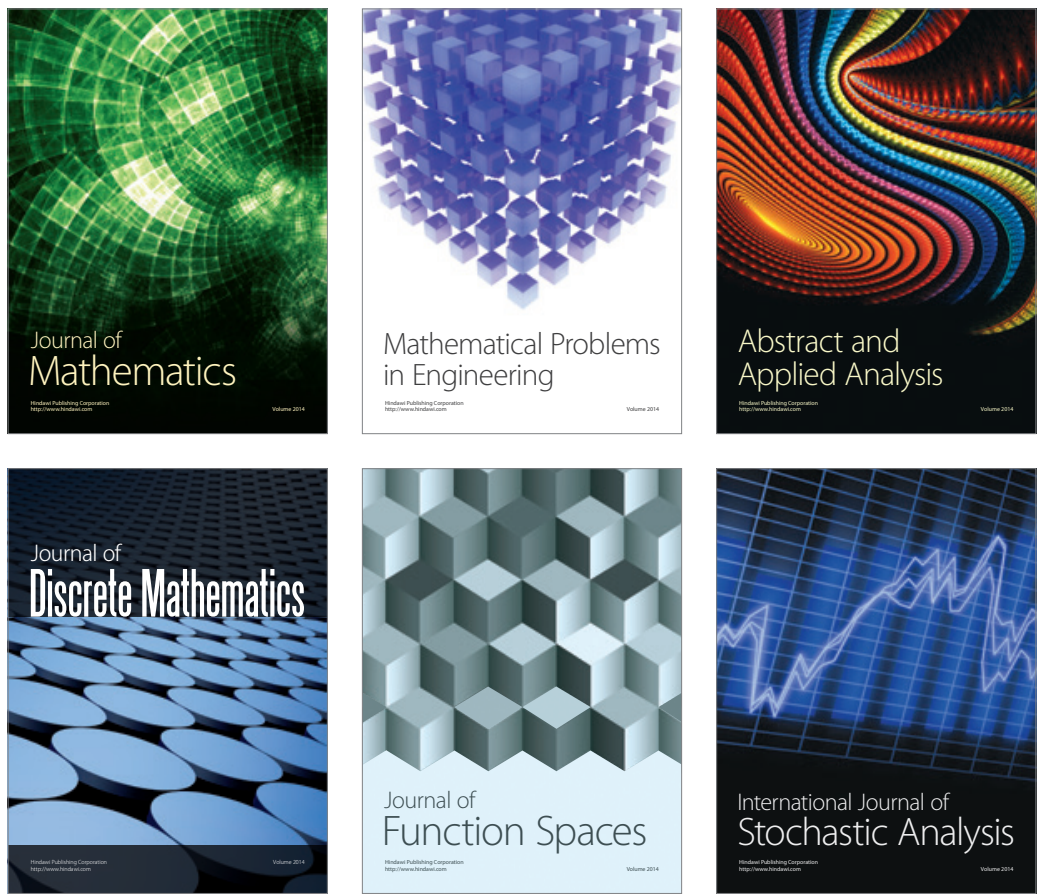

Journal of

Function Spaces

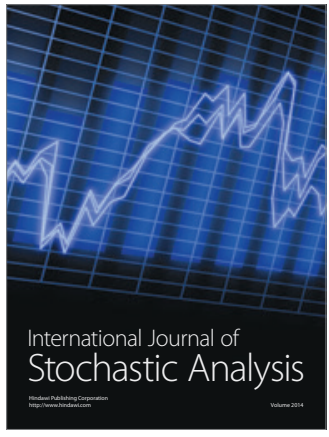

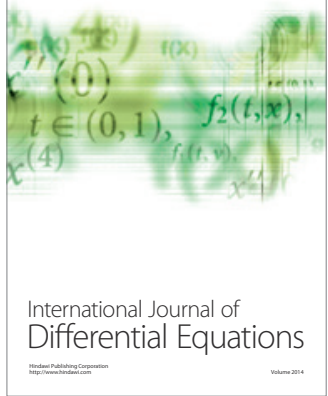
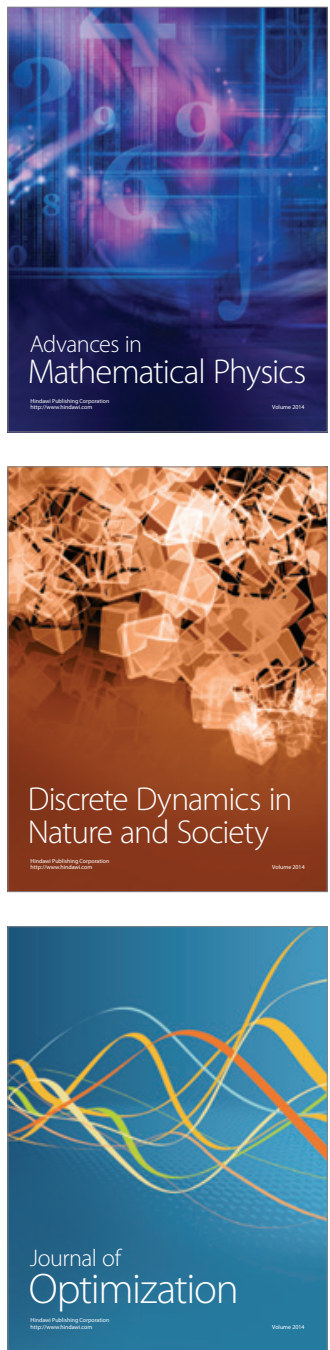